\title{
The impact of chronic rhinosinusitis on sleep-disordered breathing*
}

\author{
Rong-San Jiang ${ }^{1,2,5}$, Kai-Li Liang ${ }^{1,2,5}$, Chung-Han Hsin ${ }^{2,4}$, Mao-Chang Su',3,4 \\ Department of Otolaryngology, Taichung Veterans General Hospital, Taichung, Taiwan \\ ${ }_{2}^{2}$ School of Medicine, Chung Shan Medical University, Taichung, Taiwan \\ ${ }^{3}$ School of Speech Language Pathology \& Audiology, Chung Shan Medical University, Taichung, Taiwan \\ ${ }^{4}$ Department of Otolaryngology, Chung Shan Medical University Hospital, Taichung, Taiwan \\ ${ }^{5}$ Faculty of Medicine, National Yang-Ming Medical University, Taipei, Taiwan
}

Rhinology 54: 75-79, 2016

DOI:10.4193/Rhino15.204

*Received for publication:

July 16, 2014

Accepted: August 27, 2015

\begin{abstract}
Background: The nose plays an important role in sleep quality. Very little is known about sleep problems in patients with chronic rhinosinusitis (CRS). The aim of this study was to investigate the impact of CRS on sleep-disordered breathing.

Methodology: CRS patients who underwent functional endoscopic sinus surgery were collected between July 2010 and May 2015. Before surgery, they filled 20-item Sino-Nasal Outcome Test and Epworth Sleepiness Scale questionnaires, were asked about the severity of nasal obstruction, and received acoustic rhinometry, smell test, an endoscopic examination, sinus computed tomography, and a one-night polysomnography. Sleep quality was evaluated in these patients and was correlated with the severity of rhinosinusitis.
\end{abstract}

Results: One hundred and thirty-nine CRS patients were enrolled in the study. Among them, 38.1\% complained of daytime sleepiness, and this sleep problem was correlated with the symptom of nasal obstruction. Obstructive sleep apnea syndrome (OSAS) was diagnosed in $64.7 \%$ of the patients, but there was no correlation with the severity of rhinosinusitis. Nasal polyps did not worsen sleep problems in the CRS patients.

Conclusions: This study showed that CRS patients had a high prevalence of OSAS, and worse OSAS in CRS patients was not correlated with the severity of rhinosinusitis.

Key words: nasal obstruction, nasal polyps, obstructive sleep apnea syndrome, polysomnography, sinusitis, sleep

\section{Introduction}

Sleep-disordered breathing is a spectrum of breathing abnormalities related to increased airway resistance. It includes snoring, upper airway resistance syndrome, and obstructive sleep apnea syndrome (OSAS) ${ }^{(1)}$. The nose has been shown to play an important role in the pathophysiology of sleep-disordered breathing ${ }^{(2)}$. Allergic rhinitis was shown to be an independent risk factor for obstructive sleep apnea syndrome (OSAS) ${ }^{(3)}$. It is thought that nasal obstruction causes increased airway resistance resulting in the development of OSAS in these patients. Chronic rhinosinusitis (CRS) is a very common sinonasal disorder, with up to $13 \%$ of American adults affected ${ }^{(4)}$. Sleep impairment has been reported to be a common problem in CRS patients ${ }^{(5)}$. They also often complain of nasal obstruction which may play a role in sleep impairment in these patients. Moreover, it has been suggested that CRS is associated with the release of proinflammatory cytokines such as IL-1 $\beta$ and THF- $\alpha$ which might be involved in the development of sleep problems ${ }^{(6)}$. Recently, Alt et al. reported a high prevalence of poor quality of sleep in CRS patients, as measured by the Pittsburg Sleep Quality Index instrument ${ }^{(7)}$. However, whether sleep impairment in CRS patients is related to increased airway resistance is not well studied.

The impact of allergic rhinitis, CRS, and nasal polyposis on sleep has been evaluated by subjective and objective measures ${ }^{(5)}$. CRS 
disease-specific questionnaires such as the Sino-Nasal Outcome Test-20 (SNOT-20), which includes sleep-specific survey items, have been used to evaluate sleep and its role in CRS ${ }^{(6)}$. General sleep questionnaires such as the Epworth Sleepiness Scale (ESS) have not been widely used to evaluate sleep problems in CRS patients ${ }^{(6)}$. Moreover, relatively few studies have used objective measures such as polysomnography (PSG) to evaluate sleep problems in CRS patients ${ }^{(8)}$. The aims of this study were to explore the relationship of CRS and sleep-disordered breathing by using the SNOT-20, ESS, and PSG.

\section{Materials and methods}

\section{Subjects}

CRS patients who responded poorly to medical treatment and subsequently underwent functional endoscopic sinus surgery (FESS) were collected in this study between July 2010 and May 2015. The diagnosis of CRS was based on guidelines of the SAHP task force in 2002, which included a history of rhinosinusitis for more than 12 weeks, the findings of nasal endoscopy, and a radiological examination ${ }^{(9)}$. Any patient whose age was below 20 years old or who had a history of immunodeficiency or a previous sinus surgery was not enrolled. This study was approved by the Ethics Committee of Taichung Veterans General Hospital.

\section{Assessment of rhinosinusitis severity}

Before the FESS procedure, all patients completed the Taiwanese version of the 20-item Sino-Nasal Outcome Test (SNOT-20) questionnaire ${ }^{(10)}$, and were asked about the severity of nasal obstruction. Patients also received an acoustic rhinometry assessment, two smell tests, an endoscopic examination, and sinus computed tomography (CT).

SNOT-20 is a 20-item questionnaire which is used to assess the degree and effect of treating patients with rhinitis and rhinosinusitis. Based on the frequency and severity of symptoms, the patient scores each question from zero to five ( 0 for no problem, 5 for the problem as bad as it can be). The total score is the sum of the scores of all the items (range 0-100). The severity of nasal obstruction was also graded from zero to five, as in the SNOT-20. Acoustic rhinometry was performed to measure the second minimal cross-sectional area (MCA2) of the nasal cavity. The MCA2 of the right and left nasal cavity was averaged to give a mean MCA2 $\left(\mathrm{cm}^{2}\right)$. The smell function was evaluated by Smell Threshold Test (Sensonics, Inc., Hadden Heights, NJ) and a traditional Chinese version of the University of Pennsylvania Smell Identification Test (UPSIT-TC) (Sensonics, Inc., Hadden Heights, NJ) ${ }^{(11)}$. The endoscopic appearances were quantified on a 0 to 2-point scale according to the staging system devised by Lund and Mackay ${ }^{(12)}$. Endoscopic appearances included the presence of polyps, discharge, and edema. The total score is the sum of the all the scores of the bilateral endoscopic findings (range 0-12). Based on the endoscopic findings, patients were
Table 1. Clinical characteristics when classified by ESS score.

\begin{tabular}{|c|c|c|c|}
\hline ESS score & Below 10 & 10 or more & p value \\
\hline Gender & & & 0.045 \\
\hline Male & 51 & 41 & \\
\hline Female & 35 & 12 & \\
\hline Age (years) & $46.0 \pm 14.4$ & $42.8 \pm 12.2$ & 0.241 \\
\hline BMI & $25.01 \pm 4.22$ & $25.57 \pm 4.06$ & 0.41 \\
\hline ESS score & $5.6 \pm 2.8$ & $13.4 \pm 3.1$ & $<0.001$ \\
\hline $\mathrm{AHI}$ & $14.44 \pm 17.67$ & $16.16 \pm 19.69$ & 0.609 \\
\hline Lowest $\mathrm{SaO}_{2}(\%)$ & $86.62 \pm 7.14$ & $85.38 \pm 7.59$ & 0.205 \\
\hline Snoring index & $248.90 \pm 165.02$ & $276.72 \pm 160.87$ & 0.34 \\
\hline SNOT-20 & $33.8 \pm 19.7$ & $43.8 \pm 17.3$ & 0.003 \\
\hline Sleep-related score & $13.3 \pm 9.6$ & $16.6 \pm 8.1$ & 0.016 \\
\hline Nasal obstruction & $2.9 \pm 1.6$ & $3.6 \pm 1.4$ & 0.009 \\
\hline $\mathrm{MCA}_{2}$ & $0.43 \pm 0.22$ & $0.41 \pm 0.19$ & 0.885 \\
\hline Smell threshold & $-3.86 \pm 3.27$ & $-3.52 \pm 3.04$ & 1 \\
\hline UPSIT-TC & $20.6 \pm 9.3$ & $21.1 \pm 9.2$ & 0.715 \\
\hline Endoscopy & $5.2 \pm 2.5$ & $5.2 \pm 2.2$ & 0.969 \\
\hline $\mathrm{CT}$ & $11.2 \pm 5.2$ & $12.3 \pm 5.0$ & 0.854 \\
\hline
\end{tabular}

BMI: body mass index; ESS: Epworth Sleepiness Scale; AHI: apneahypopnea index; $\mathrm{SaO}_{2}$ : oxygen saturation; SNOT-20: 20-item Sino-Nasal Outcome Test; MCA $_{2}$ : the second minimal cross-sectional area; UPSITTC: traditional Chinese version of the University of Pennsylvania Smell Identification Test; CT: computed tomography.

divided into those with and without nasal polyps. The sinus CT scan findings were quantified according to the staging method described by Lund and Mackay ${ }^{(12)}$. The CT findings of maxillary, anterior ethmoid, posterior ethmoid, sphenoid, and frontal sinus were graded as 0,1 , or 2 . The total score is the sum of all the scores of the bilateral sinuses (range $0-24$ ).

\section{Assessment of sleep quality}

Before the FESS procedure, all patients also completed a Chinese version of the ESS questionnaire ${ }^{(13)}$, and received a one-night PSG. Sleep quality was also evaluated using the 7 sleep-related items of the SNOT-20. The 7 sleep-related items were item 11: difficulty falling asleep, item 12: waking up at night, item 13: lack of a good night's sleep, item 14: waking up tired, item 15: fatigue, item 16: reduced productivity, and item 17: reduced concentration.

ESS is an 8-item questionnaire which is a useful tool for evaluating daytime sleepiness in adults ${ }^{(13)}$. Each of the 8-item scores ranges from 0 to 3 , and the total ESS score is from 0 to 24 . A score of 10 or more is considered to indicate that the patient 
Table 2. Clinical characteristics when classified by AHI.

\begin{tabular}{|c|c|c|c|c|c|}
\hline ESS score & Without OSAS & Mild OSAS & Moderate OSAS & Severe OSAS & p value \\
\hline Gender & & & & & 0.585 \\
\hline Male & 34 & 25 & 24 & 12 & \\
\hline Female & 18 & 16 & 8 & 5 & \\
\hline Age (years) & $39.4 \pm 11.9$ & $43.7 \pm 12.5$ & $51.6 \pm 15.0$ & $49.5 \pm 11.8$ & 0.068 \\
\hline BMI & $23.88 \pm 4.16$ & $25.49 \pm 3.15$ & $24.66 \pm 3.39$ & $29.52 \pm 4.87$ & 0.003 \\
\hline ESS score & $8.2 \pm 4.9$ & $9.0 \pm 4.6$ & $8.6 \pm 4.6$ & $8.3 \pm 5.3$ & 0.758 \\
\hline $\mathrm{AHI}$ & $1.96 \pm 1.30$ & $9.01 \pm 2.76$ & $20.35 \pm 4.31$ & $57.74 \pm 15.97$ & $<0.001$ \\
\hline Lowest $\mathrm{SaO}_{2}(\%)$ & $89.96 \pm 3.69$ & $87.02 \pm 4.88$ & $84.83 \pm 6.31$ & $75.48 \pm 10.63$ & $<0.001$ \\
\hline Snoring index & $128.27 \pm 118.36$ & $271.84 \pm 107.83$ & $323.55 \pm 114.93$ & $487.46 \pm 130.89$ & $<0.001$ \\
\hline SNOT-20 & $42.1 \pm 19.6$ & $39.7 \pm 18.3$ & $31.0 \pm 19.8$ & $32.0 \pm 17.3$ & 0.094 \\
\hline Sleep-related score & $16.6 \pm 9.5$ & $15.4 \pm 8.9$ & $11.2 \pm 8.3$ & $13.2 \pm 9.0$ & 0.13 \\
\hline Nasal obstruction & $3.1 \pm 1.5$ & $3.6 \pm 1.3$ & $2.9 \pm 1.7$ & $2.9 \pm 1.6$ & 0.104 \\
\hline $\mathrm{MCA}_{2}$ & $0.39 \pm 0.2$ & $0.43 \pm 0.2$ & $0.40 \pm 0.2$ & $0.53 \pm 0.27$ & 0.165 \\
\hline Smell threshold & $-4.60 \pm 3.44$ & $-3.33 \pm 2.82$ & $-3.86 \pm 3.42$ & $-1.96 \pm 1.68$ & 0.151 \\
\hline UPSIT-TC & $22.8 \pm 9.8$ & $19.7 \pm 8.3$ & $21.0 \pm 9.5$ & $17.3 \pm 8.3$ & 0.543 \\
\hline Endoscopy & $4.8 \pm 2.0$ & $5.5 \pm 2.5$ & $5.4 \pm 2.5$ & $5.5 \pm 2.9$ & 0.992 \\
\hline СT & $10.9 \pm 4.6$ & $12.0 \pm 5.1$ & $12.3 \pm 5.7$ & $11.3 \pm 6.0$ & 0.848 \\
\hline
\end{tabular}

AHI: apnea-hypopnea index; OSAS: obstructive sleep apnea syndrome; BMI: body mass index; ESS: Epworth Sleepiness $\mathrm{Scale}_{\text {; }} \mathrm{SaO}_{2}$ : oxygen saturation; SNOT-20: 20-item Sino-Nasal Outcome Test; MCA $_{2}$ : the second minimal cross-sectional area; UPSIT-TC: traditional Chinese version of the University of Pennsylvania Smell Identification Test; CT: computed tomography.

suffers from daytime sleepiness ${ }^{(14)}$. Therefore, patients were divided into those whose ESS scores were below 10 and whose ESS scores were 10 or more. PSG has been used as a reference standard for the diagnosis of OSAS ${ }^{(15)}$. It measures several sleep variables, including body mass index (BMI), apnea-hypopnea index $(\mathrm{AHI})$ or respiratory disturbance index, and minimum oxygen saturation. The $\mathrm{AHI}$ is defined as the sum of apneas and hypopneas per hour of sleep. Apnea is defined as a $90 \%$ decrease in airflow for 10 seconds relative to the baseline value. Hypopnea is defined as a $50 \%$ decrease in the airflow amplitude for 10 seconds relative to the baseline value with the presence of arousal or oxygen desaturation of $4 \%$. Patients with AHIs of $\geq 5$ and $<15$ were considered to have mild OSAS, those with AHIs of $\geq 15$ and $<30$ were considered to have moderate OSAS, and those with AHIs of $\geq 30$ were considered to have severe OSAS ${ }^{(8)}$. According to the severity of OSAS, patients were divided into those without OSAS (AHI <5), those with mild OSAS, those with moderate OSAS, and those with severe OSAS.

\section{Statistical analysis}

All data are presented as mean \pm standard deviation. Patients were divided into 2 ESS groups based on ESS score (below 10 and 10 or more), into $4 \mathrm{AHI}$ groups based on $\mathrm{AHI}$ value $(<5, \geq 5$ and $<15, \geq 15$ and $<30$, and $\geq 30$ ), and into 2 polyp groups based on the existence of nasal polyps (with and without polyps). The severity of sinusitis was compared between the 2 ESS groups and among the $4 \mathrm{AHI}$ groups, and sleep quality was compared between the 2 polyp groups. The sex of patients was compared between the 2 ESS groups, among the $4 \mathrm{AHI}$ groups, and between the 2 polyp groups by Pearson's Chi-Square test. The age of patients, SNOT-20 score, total score of 7 sleep-related items, nasal obstruction score, mean $\mathrm{MCA}_{2}$, smell threshold, UPSIT-TC score, endoscopic score, and CT score were compared between the 2 ESS groups and between the 2 polyp groups by Mann-Whitney $\mathrm{U}$ test and were compared among the $4 \mathrm{AHI}$ groups by Kruskal-Wallis test. The correlations between ESS score and SNOT-20 score, nasal obstruction score, UPSIT-TC score, endoscopic score, and CT score and the correlations between AHI value and SNOT-20 score, nasal obstruction score, UPSIT-TC score, endoscopic score, and CT score were evaluated by Spearman's correlation coefficient. All computations were performed using SPSS version 17.0 (SPSS, Inc., Chicago, IL, USA). Two-tailed p-values $<0.05$ were considered statistically significant. 


\section{Results}

\section{Patients}

One hundred and thirty-nine CRS patients were enrolled in the study. Among them, there were 92 males and 47 females. The ages ranged from 20 to 84 years old with a mean of 44.7 years. Patients' characteristics among the different groups are shown in Tables 1-3. The ESS score was below 10 in 86 patients and was 10 or more in 53 . Therefore, $38.1 \%$ of patients were considered to suffer from daytime sleepiness. Forty-nine patients were without OSAS, 41 had mild OSAS, 32 had moderate OSAS, and 17 had severe OSAS. In total, $64.7 \%$ of patients were diagnosed with OSAS. Seventy patients were with nasal polyps and 69 were without polyps.

Comparison of rhinosinusitis severity between ESS groups The comparison of rhinosinusitis severity and sleep quality between the 2 ESS Groups is shown in Table 1. Patients with poor sleep quality ( $E S S \geq 10$ ) had significantly higher SNOT-20 and nasal obstruction scores, but the endoscopic score, CT score and smell function were not significantly different between the 2 ESS groups. The correlation analysis also showed a positive correlation between ESS score and SNOT-20 score $(r=0.306$; $p<0.001)$ as well as nasal obstruction score $(r=0.297 ; p<0.001)$, but there was no correlation between ESS score and UPSIT-TC score, endoscopic score, and CT score.

Comparison of rhinosinusitis severity among AHI groups The comparison of rhinosinusitis severity and sleep quality among the $4 \mathrm{AHI}$ groups is shown in Table 2. The SNOT-20 score, nasal obstruction score, endoscopic score, CT score, and smell function were not significantly different among the $4 \mathrm{AHI}$ groups. The correlation analysis also showed there was no correlation between ESS score and SNOT-20 score, nasal obstruction score, UPSIT-TC score, endoscopic score, and CT score.

Comparison of sleep quality between polyp groups The comparison of sleep quality and rhinosinusitis severity between the 2 polyp groups is shown in Table 3 . Rhinosinusitis was more severe in patients with nasal polyps than in those without polyp when assessed by nasal obstruction score, mean MCA2, smell threshold, UPSIT-TC score, endoscopic score, and CT score. However, sleep quality was similar between patients with and without nasal polyps.

\section{Discussion}

Sleep problems have not been well studied in CRS patients ${ }^{(5)}$. Alt et al. reported that $75 \%$ of CRS patients have PSQI scores above the traditional cutoff, indicating poor sleep quality ${ }^{(7)}$. Among our patients, $38.1 \%$ reported ESS scores above 9, indicating daytime sleepiness. The patients with poor sleep quality in the study by Alt et al. had significantly higher SNOT-22 scores
Table 3. Weighted percentages by city, age and gender of children/ adolescents characteristics and OR of the association between possible related factors and $A R$.

\begin{tabular}{|lccc|}
\hline Nasal polyps & $\begin{array}{c}\text { Without } \\
\text { polyps }\end{array}$ & With polyps & p value \\
\hline Gender & & & 0.952 \\
\hline \multicolumn{1}{|c}{ Male } & 45 & 47 & \\
\hline \multicolumn{1}{|c}{ Female } & 24 & 23 & \\
\hline Age (years) & $44.8 \pm 13.1$ & $44.6 \pm 14.2$ & 0.889 \\
\hline BMI & $25.10 \pm 3.95$ & $25.35 \pm 4.36$ & 0.588 \\
\hline ESS score & $8.9 \pm 4.6$ & $8.3 \pm 4.9$ & 0.499 \\
\hline AHI & $14.17 \pm 18.98$ & $16.02 \pm 17.93$ & 0.26 \\
\hline Lowest SaO ${ }_{2}(\%)$ & $86.23 \pm 7.92$ & $86.05 \pm 6.71$ & 0.412 \\
\hline Snoring index & $241.80 \pm 172.49$ & $276.96 \pm 153.21$ & 0.153 \\
\hline SNOT-20 & $40.6 \pm 19.4$ & $34.7 \pm 19.0$ & 0.066 \\
\hline Sleep-related score & $16.0 \pm 9.4$ & $13.1 \pm 8.7$ & 0.067 \\
\hline Nasal obstruction & $2.8 \pm 1.5$ & $3.5 \pm 1.5$ & 0.006 \\
\hline MCA 2 & $0.46 \pm 0.23$ & $0.38 \pm 0.19$ & 0.024 \\
\hline Smell threshold & $-4.98 \pm 3.26$ & $-2.50 \pm 2.58$ & $<0.001$ \\
\hline UPSIT-TC & $25.1 \pm 8.3$ & $16.6 \pm 8.0$ & $<0.001$ \\
\hline Endoscopy & $3.8 \pm 1.6$ & $6.6 \pm 2.2$ & $<0.001$ \\
\hline CT & $8.4 \pm 4.1$ & $14.7 \pm 4.1$ & $<0.001$ \\
\hline
\end{tabular}

BMI: body mass index; ESS: Epworth Sleepiness Scale; AHI: apneahypopnea index; $\mathrm{SaO}_{2}$ : oxygen saturation; SNOT-20: 20-item Sino-Nasal Outcome Test; $\mathrm{MCA}_{2}$ : the second minimal cross-sectional area; UPSITTC: traditional Chinese version of the University of Pennsylvania Smell Identification Test; CT: computed tomography.

compared with those of patients with good sleep quality, but there were no significant differences in endoscopic, $\mathrm{CT}$, or smell function scores between the two groups ${ }^{(7)}$. The patients with poor sleep quality $(E S S \geq 10)$ in our study also had significantly higher SNOT-20 scores than those of patients with good sleep quality, but there were no differences in endoscopic, CT, or smell function scores between the two groups. Among the patients in the study by Alt et al. women had a higher prevalence of poor sleep quality than men ${ }^{(7)}$, but in the present study men had a higher prevalence of poor sleep quality than women. The etiology of poor sleep quality in CRS patients is likely multifactorial. Our patients with poor sleep quality had significantly higher nasal obstruction scores, and ESS score was positively correlated with nasal obstruction scores. This indicates that nasal obstruction plays an important role in the pathophysiology of sleep impairment in CRS patients. However, other factors might also play a 
role such as cytokines ${ }^{(6)}$.

OSAS is a very common disorder, afflicting $\mathbf{4 0}$ million Americans (4). Its prevalence is increasing, and approximately $24 \%$ of men and $9 \%$ of women are affected. In this study, $66.3 \%$ of male patients and $61.7 \%$ of female patients were diagnosed with OSAS (AHI $\geq 5$ ). Our CRS patients were unresponsive to medical treatment, which suggests that symptomatic CRS patients, regardless of gender, had a higher prevalence of OSAS than that in the general population. Nasal obstruction and inflammation of nasal airway mucosa have been suggested to be possible etiologies in the pathogenesis of OSAS in CRS patients ${ }^{(8)}$. The high prevalence of OSAS in CRS patients was unrelated to BMI. Although BMI of patients with severe OSAS was higher than that of patients without OSAS, BMI of patients without OSAS was not significantly different from those of patients with mild or moderate OSAS ( $p=0.266,0.846$, respectively). The worsening of OSAS in CRS patients was also not correlated with the severity of rhinosinusitis. The SNOT-20 score, nasal obstruction score, endoscopic score, CT score, and smell function were not significantly different among the $4 \mathrm{AHI}$ groups.

It has been reported that patients with nasal polyps had a higher risk of sleep impairment and a greater prevalence of snoring than controls without nasal polyps ${ }^{(16)}$. When our CRS patients were classified into those with and without nasal polyps, the severity of rhinosinusitis was found to be significantly higher in CRS patients with nasal polyps than in those without nasal polyps, and sleep quality whether evaluated by ESS or PSG was not significantly different between CRS patients with and without nasal polyps. This result indicates that nasal polyps did not worsen sleep problem in CRS patients, and the severity of rhinosinusitis also did not have a deleterious effect on sleep quality in CRS patients.

\section{Conclusion}

The results of this study showed that $38 \%$ of CRS patients suffered from daytime sleepiness, and this sleep problem was related to the symptom of nasal obstruction, which was very common in CRS patients. CRS patents had a higher prevalence of OSAS. The worsening of OSAS in CRS patients was not correlated with the severity of rhinosinusitis, and presence of nasal polyps did not appear to be associated with worse sleep problems in CRS patients.

\section{Authorship contribution}

RSJ: study design, data collection, manuscript; KLL: study design, data analysis; $\mathrm{HCH}$ : manuscript edits; SMC: study design, manuscript edits.

\section{Conflict of interest}

The authors declare no conflict of interest.

\section{References}

1. Rappai M, Collop N, Kemp S, deShazo R. The nose and sleep-disordered breathing: what we know and what we do not know. Chest. 2003; 124: 2309-2323.

2. Meen EK, Chandra RK. The role of the nose in sleep-disordered breathing. Am J Rhinol Allergy. $2013 ; 27: 213-220$

3. Acar M, Cingi C, Sakallioglu $O$, et al. The effects of mometasone furoate and desloratadine in obstructive sleep apnea syndrome patients with allergic rhinitis. Am J Rhinol Allergy. 2013; 27: e113-116.

4. Lachanas VA, Woodard TD, Antisdel JL, Kountakis SE. Sino-nasal outcome test too assessment in patients with chronic rhinosinusitis and obstructive sleep apnea ORL J Otorhinolaryngol Relat Spec. 2012; 74: 286-289.

5. Craig TJ, Ferguson BJ, Krouse JH. Sleep impairment in allergic rhinitis, rhinosinusitis, and nasal polyposis. Am J Otolaryngol. 2008; 29: 209-217.

6. Alt JA, Smith TL. Chronic rhinosinusitis and sleep: a contemporary review. Int Forum Allergy Rhinol. 2013; 3: 941-949.

7. Alt JA, Smith TL, Mace JC, Soler ZM. Sleep quality and disease severity in patients with chronic rhinosinusitis. Laryngoscope. 2013; 123: $2364-2370$

8. Yalamanchali S, Cipta S, Waxman J, Pott $T$, Joseph N, Friedman M. Effects of
Endoscopic Sinus Surgery and Nasal Surgery in Patients with Obstructive Sleep Apnea. Otolaryngol Head Neck Surg. 2014; 151: 171-175.

9. Benninger MS, Ferguson BJ, Haley JA, et al. Adult chronic rhinosinusitis: definitions, diagnosis, epidemiology, and pathophysiology. Otolaryngol Head Neck Surg. 2003; 129(3 Suppl): S1-S32.

10. Liang KL, Twu CW, Shiao JY, et al. Validation of the Taiwanese version of the 20-item Sino-nasal Outcome Test. J Taiwan Otolaryngol Head Neck Surg. 2011; 46: 6-13.

11. Jiang RS, Su MC, Liang KL, Shiao JY, Wu $\mathrm{SH}, \mathrm{H} \sin \mathrm{CH}$. A pilot study of a traditional Chinese version of the University of Pennsylvania Smell Identification Test for application in Taiwan. Am J Rhinol Allergy. 2010; 24: 45-50.

12. Lund VJ, Mackay IS. Staging in rhinosinusitis. Rhinology. 1993; 31: 183-184.

13. Chen NH, Johns MW, Li HY, et al. Validation of a Chinese version of the Epworth sleepiness scale. Qual Life Res. 2002; 11: 817-821.

14. Liu YF, Hu J, Streelman M, Guthrie OW. The Epworth sleepiness scale in the assessment of sleep disturbance in veterans with tinnitus. Int J Otolaryngol. 2015; 2015: 429469.

15. Health Quality Ontario. Polysomnography in patients with obstructive sleep apnea: an evidence-based analysis. Ont Health Technol Assess Ser. 2006; 6: 1-38.
16. Serrano E, Neukirch F, Pribil C, et al. Nasal polyposis in France: impact on sleep and quality of life. J Laryngol Otol. 2005; 119: 543-549.

Mao-Chang Su, M.D., Ph.D

Department of Otolaryngology

Chung Shan Medical University

Hospital

110, Section 1, Chien-Kuo N. Road,

Taichung 40201

Taiwan

Tel.: +886-4-23592525 ext. 5401

Fax: +886-4-23596868

E-mail: smc@csmu.edu.tw 